\title{
Image Enhancement System Based on Improved Dark Channel Prior
}

\author{
Chang Liu ${ }^{1, a}$, Jun Zhu ${ }^{1, b}$ and Xiaojun Peng ${ }^{1, c}$ \\ ${ }^{1}$ Wuhan Second Ship Design and Research Institute,Wuhan 430064,China \\ aimliuchang@outlook.com, b'zhujun.139@139.com, ckingarthurpeng@hotmail.com
}

Keywords: Image enhancement, dark channel prior, Image dehazing, DM6467, Image evaluation. Abstract. In order to reduce the bad influence of hazes on the computer vision system, an image dehazing algorithm based on dark channel prior is proposed. Firstly, we analyze the dark channel prior based image dehazing algorithm. Since the complexity of the algorithm is great and the acquired images have halo artifacts effect, the neighborhood similarity method, which gets the difference of the value of dark channel between every pixel and its nearest eight pixels, is introduced. The pixel of minimal difference is redefined as new dark channel. Besides, the adaptive gray stretch and local contrast enhancement algorithms are proposed to increase brightness and definition of the video. Finally, realization of the improved image enhancement algorithms based on DM6467 is analyzed in detail. The results indicate that the method can effectively improve the image quality.

\section{Introduction}

Computer vision system has been widely used in all aspects of social production and daily life, such as urban transportation, security facilities, video monitoring, etc. However, in foggy weather, the scene light is absorbed or scattered by the atmosphere with a large number of suspended particles (dust particles and water droplets, etc.).Camera image quality deteriorates with poor visibility and color distortion, which greatly affects the reliability and robustness of computer vision system.

Image dehazing is a hotspot in the fields of computer vision and image processing. He et al. [1] proposed a simple but effective image prior-dark channel prior to remove haze from a single input image, which can achieve great haze removal effect. The method has been widely in image processing field $[2,3]$.

However, as it is based on the assumption that the transmission is locally constant, the patch size will affect the quality of dehazed images [4]. A large patch size leads to bright atmosphere but serious halo artifacts, while a small one can achieve nice dehazing results with little halo artifacts but dim atmosphere. And the process of optimizing the medium transmission in the improved algorithm $[5,6]$ costs too much time, while the computational complexity is too high to be real-time operating for high resolution image.

In this paper, an improved dark channel prior based image dehazing algorithm is designed by introducing neighborhood similarity method to reduce halo artifacts without soft matting. In order to improve image quality after image dehazing, some image enhancement algorithms are introduced, such as linear gray expanding and local contrast enhancement. Then, an image enhancement system is developed by applying the image processing algorithms based on the TI's DaVinci technology. The system meets the real-time and good interaction demands.

The rest of the paper is organized as follows. Section 2 describes the video image enhancement architecture. In Section 3, the proposed method for image enhancement is detailed. Experimental results on image enhancement performance are illustrated in Section 4 followed by the conclusions in Section 5 .

\section{The Video Image Enhancement Architecture}

The video image enhancement system sharpens hazy images based on TMS320DM6467 (also referenced as DM6467) Digital Media System-on-Chip with image processing algorithms porting in the Linux Embedded Operating System.

The DM6467 leverages TI's DaVinci technology to meet the networked media encode and decode digital media processing needs of next-generation embedded devices. The dual-core architecture of 
the DM6467 provides benefits of both DSP and Reduced Instruction Set Computer (RISC) technologies, incorporating a high-performance TMS320C64x+ DSP core and anARM926EJ-S core.

The image enhancement algorithms are based on DSP/BIOS of DSP, while the peripheral equipments are managed by MontaVista Linux of ARM. The data interaction between ARM and DSP is realized by Codec Engine and Codec Server.

The video image enhancement system is composed of three parts: front-end image acquisition equipment, image processing unit DM6467 and man-machine interactive interface. Two TVP5158 chips are extended based on DM6467, which can realize 8 channel image collections. The image processing, encoding and storage are carried out in the image processing unit. The system has good human-computer interface by introducing the simple mouse operation.

The general schematic view of video image enhancement system is shown in Fig. 1.

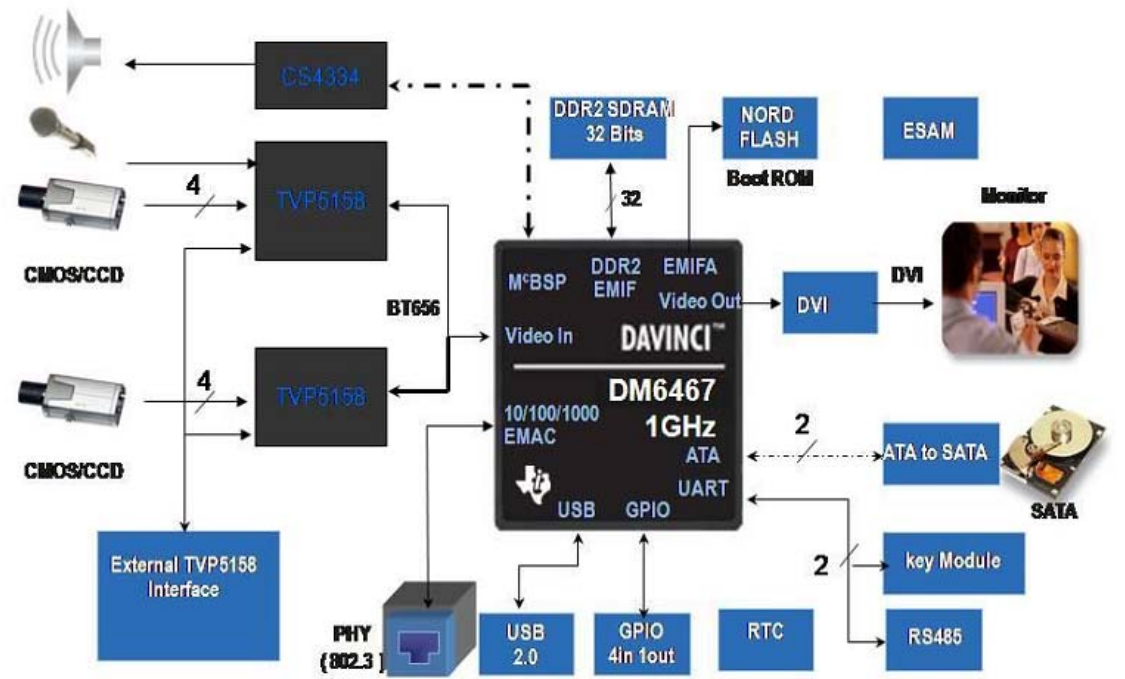

Fig. 1.The general schematic view of video image enhancement system

\section{Image Enhancement Algorithm}

Image dehazing.In the fields of computer vision and graphics, McCarney atmospheric scattering model is widely used to describe the image information of frog, which is shown in Eq. 1.

$$
I(x)=J(x) t(x)+(1-t(x)) A .
$$

WhereI(x) and $\mathrm{J}(\mathrm{x})$ denote the observed image and original image, $A$ is the global atmospheric light, $t(x)$ is the transmission of the light reflected by the object. The purpose of image dehazing is to recover the original image, global atmospheric light and transmission from the observed image.

In order to estimate $t(x)$ from single image directly, He [1] proposed dark channel prior. Statistics of numerous images show that at least one color channel has very low intensity at some pixels in local area of a single image.

$$
\mathrm{J}^{\mathrm{dark}}(\mathrm{x})=\min _{\mathrm{c} \in\{\mathrm{r}, \mathrm{g}, \mathrm{b}\}}\left(\min _{\mathrm{y} \in \Omega(\mathrm{x})}\left(\mathrm{J}^{\mathrm{c}}(\mathrm{y})\right)\right) \text {. }
$$

Where $\mathrm{c} \in\{\mathrm{r}, \mathrm{g}, \mathrm{b}\}$ is one of three color channels $\mathrm{R}, \mathrm{G}, \mathrm{B} . \Omega(\mathrm{x})$ is a local patch centered at pixel $\mathrm{x}$. $\mathrm{J}^{\text {dark }}(\mathrm{x})$ always tends to be zero in haze-free image, while $\mathrm{J}^{\mathrm{dark}}(\mathrm{x})$ increases in haze image.

In order to get the transmissiont(x), we assume that the atmospheric light $A$ is given and the transmission is constant in a local patch $\Omega(\mathrm{x})$. Eq. 1 can be rewritten as

$$
\min _{\mathrm{c}}\left(\min _{\mathrm{y} \in \Omega(\mathrm{x})}\left(\frac{\mathrm{I}^{\mathrm{c}}(\mathrm{y})}{\mathrm{A}^{\mathrm{c}}}\right)\right)=\tilde{\mathrm{t}}(\mathrm{x}) \min _{\mathrm{c}}\left(\min _{\mathrm{y} \in \Omega(\mathrm{x})}\left(\frac{\mathrm{J}^{\mathrm{c}}(\mathrm{y})}{\mathrm{A}^{\mathrm{c}}}\right)\right)+(1-\tilde{\mathrm{t}}(\mathrm{x})) .
$$

According to dark channel prior, Eq. 2 can be obtained by 


$$
\mathrm{J}^{\text {dark }}(\mathrm{x})=\min _{\mathrm{c}}\left(\min _{\mathrm{y} \in \Omega(\mathrm{x})}\left(\mathrm{J}^{\mathrm{c}}(\mathrm{y})\right)\right)=0 .
$$

As $\mathrm{A}^{\mathrm{c}}$ is always positive, this leads to

$$
\min _{\mathrm{c}}\left(\min _{\mathrm{y} \in \Omega(\mathrm{x})}\left(\frac{\mathrm{I}^{\mathrm{c}}(\mathrm{y})}{\mathrm{A}^{\mathrm{c}}}\right)\right)=0 .
$$

Then, the transmission $\tilde{\mathrm{t}}(\mathrm{x})$ can be simply estimated by

$$
\tilde{\mathrm{t}}(\mathrm{x})=1-\omega \min _{\mathrm{c}}\left(\min _{\mathrm{y} \in \Omega(\mathrm{x})}\left(\frac{\mathrm{I}^{\mathrm{c}}(\mathrm{y})}{\mathrm{A}^{\mathrm{c}}}\right)\right)
$$

In Eq. 6, a constant parameter $\omega$ is introduced to adjust the amount of haze for the distant objects. $\tilde{\mathrm{t}}(\mathrm{x})$ has the same value in local patch obtained by Eq. 6 . However, the transmission of every pixel is different in fact. The soft matting technique [7] can be used to make the final refined transmission map $t(x)$. Since the complexity of soft matting is great, the algorithm doesn't work real-time. Hence, the neighborhood similarity method, which gets the difference between the value of the dark channel and dark value of the nearest eight pixels, is introduced into the image dehazing algorithm.

Firstly, a moving $3 \times 3$-pixel image neighborhood window composed of every pixel's dark channel prior is constructed. The element of the $3 \times 3$ window is defined as Eq. 7 .

$$
\mathrm{w}\left(\mathrm{x}_{\mathrm{i}}\right)=\min _{\mathrm{c} \in\{\mathrm{r}, \mathrm{g}, \mathrm{b}\}}\left(\mathrm{I}^{\mathrm{c}}\left(\mathrm{x}_{\mathrm{i}}\right)\right) ; \mathrm{i}=1,2, \ldots, 9 .
$$

The central pixel's dark channel prior is compared with the other eight neighborhood pixels' dark channel prior. The dark channel prior of the pixel, which has the minimum absolute value of difference with the central pixel's dark channel prior, is chosen as new dark channel prior of the central pixel.

$$
\begin{aligned}
& \mathrm{w}=\min \left|\mathrm{w}\left(\mathrm{x}_{5}\right)-\mathrm{w}\left(\mathrm{x}_{\mathrm{i}}\right)\right| ; \mathrm{i} \in[1,4], \mathrm{i} \in[6,9] . \\
& \mathrm{I}^{\text {dark }}(\mathrm{x})=\mathrm{w}\left(\mathrm{x}_{\mathrm{i}}\right) .
\end{aligned}
$$

The 3×3-pixel image neighborhood window is moved to adjust every pixel's dark channel prior. It is obvious that the dark channel prior is similar in the area of one object by using neighborhood similarity method, while the dark channel prior is revised when the pixel belong to edge of the object.

The comparison between the traditional dark channel prior based image dehazing algorithm and our improved dark channel prior based image dehazing algorithm is shown is Fig. 2. Fig 2(b), 2(d) are the dark channel prior image and neighborhood similarity based dark channel prior image. Obviously, the traditional dark channel prior image consists of many image blocks which gray value is unique. Hence, the edge of the boat in Fig. 2(c) is not accurate, while the improved dehazing algorithm recovers the image more actually.

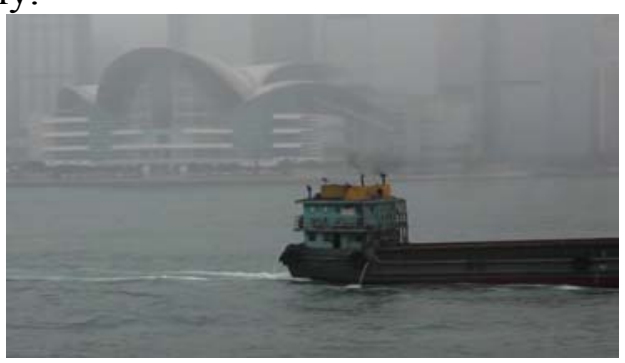

(a)Original image 


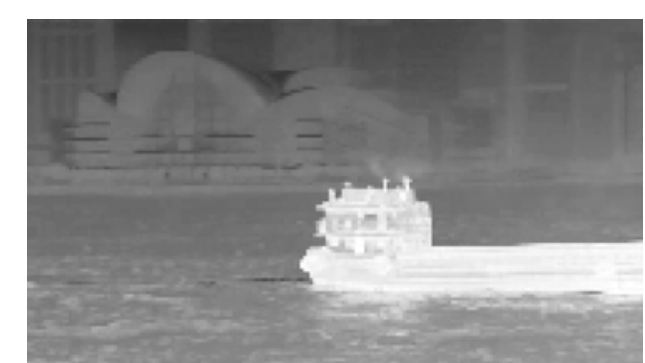

(b)The dark channel prior image

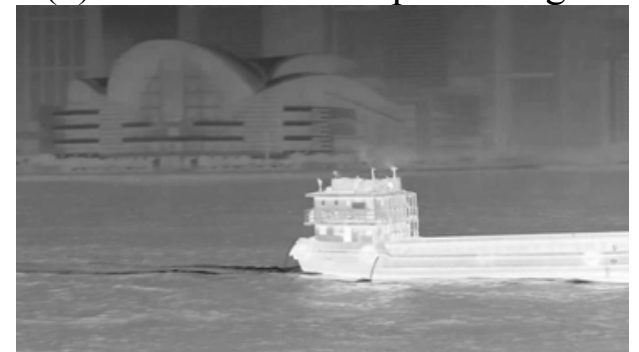

(d)Improved dark channel prior image

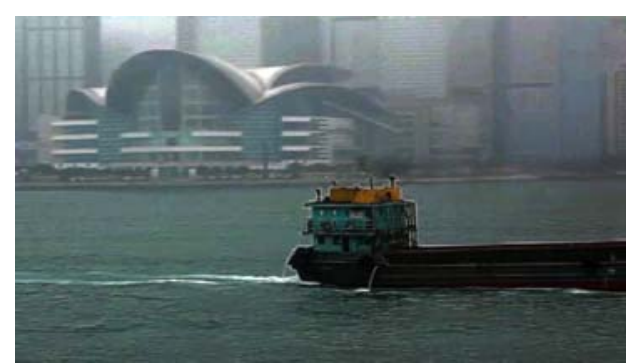

(c)Image after dark channel prior based dehazing

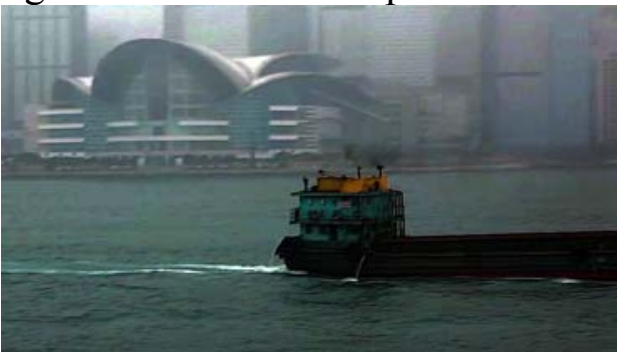

(e)Image after proposed image dehazing

Fig. 2. Comparison between dark channel prior based image dehazing and proposed image dehazing

Adaptive gray stretch.Image dehazing may lead to a loss of image quality because some images' luminance is not uniform. Additionally, the images' luminance reduces after image dehazing since the reflect light value is less than the atmosphere light. In order to improve image quality, some image enhancement algorithms are introduced, such as linear gray expanding and local contrast enhancement.

Adaptive gray stretch algorithm, by applying Histogram gray value search method to linear gray stretch algorithm, can not only increase the whole image contrast, but also improve the lightness. The algorithmic steps are as follows:

Step1: Calculate the histograms of R, G, B channels: [count1, x], [count2, x], [count3, x] respectively.

Step2: Determine the cut-off amount $\mathrm{C}$ for black_point and white_point.

Step3: Get black point: Accumulate the pixel amounts of every gray level from 0 up to 255 . Once the accumulated value reaches or exceeds thethreshold value $\mathrm{C}$, the maximum gray level is designated as black_point.

Step4: Get white_point: Accumulate the pixel amounts of every gray level from 255 down to 0. Once the accumulated value reaches or exceeds the threshold value $\mathrm{C}$, the manimum gray level is designated as white_point.

Step5: Calculate new value of every pixel by using Eq. 10.

$$
\text { hI }=(\mathrm{I} \text { - black_point }) \cdot 255 /(\text { white_point }- \text { black_point }) \text {. }
$$

In Eq. 10, I is the original pixel value, $\mathrm{hI}$ is the improvement pixel value. The value of $\mathrm{C}$ is application-based and suggested to be 5\%o of the total pixel numbers in step 2 .
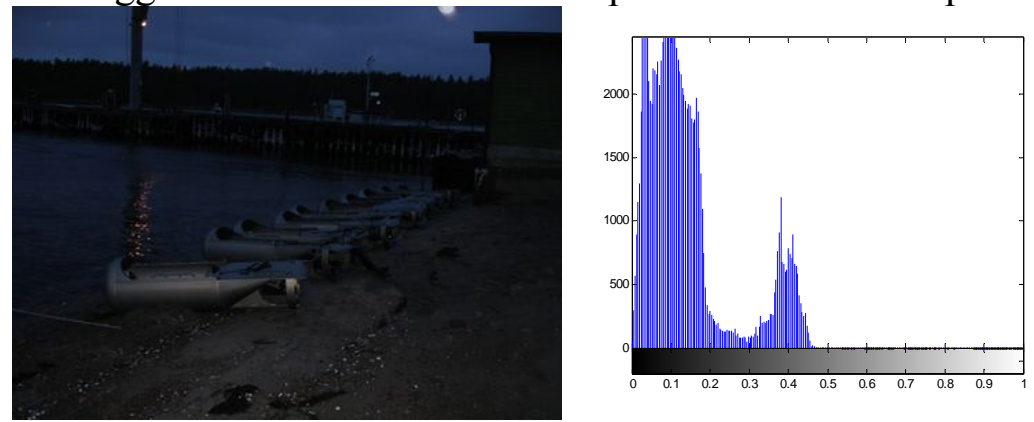

(a)Original night image and its histogram 

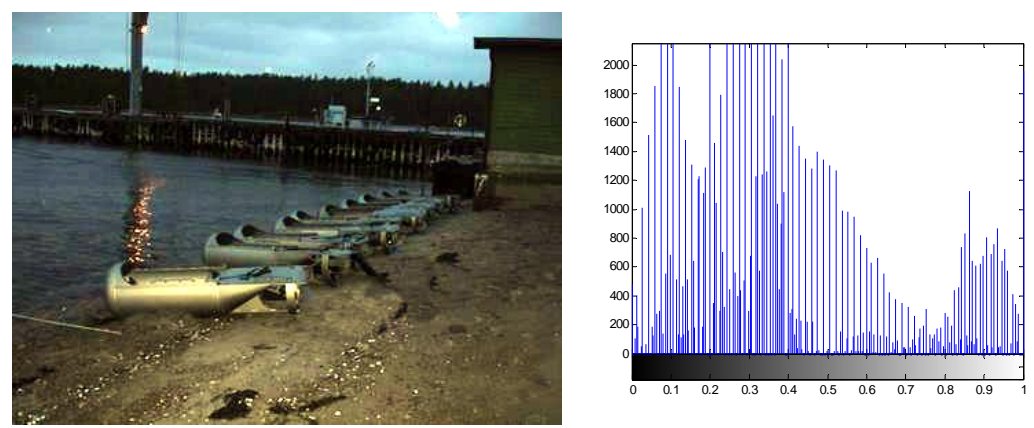

(b)Improve image after adaptive gray stretch and its histogram

Fig. 3. Comparison between a night image and the image after adaptive gray stretch

Fig. 3 shows the original image and the image after adaptive gray stretch of a night image. It is obvious that the adaptive gray stretch algorithm increases image brightness significantly.

Local contrast enhancement. Local contrast enhancement can enhance the image's minutiae feature, in other words, the definition can be improved by introducing the algorithm. The algorithmic steps are as follows:

Step1: Set a moving window $\mathrm{n} \times \mathrm{n}$ and enhancement factor $\mathrm{k}$.

Step2: Calculate the new value of the centre pixel in the $n \times n$ window by using Eq. 11 .

$\mathrm{hI}=\mathrm{m}+\mathrm{k} \cdot(\mathrm{I}-\mathrm{m})$.

Where $\mathrm{I}$ is the original pixel value, $\mathrm{hI}$ is the improvement pixel value, $\mathrm{m}$ is the arithmetic mean value of pixels in window $n \times n$.

Step3: Carry out all pixel values one after another.

The size of the window should be chosen according to the image size. It is suggested to be $3 \times 3$ or $5 \times 5$ of the image which resolution is D1. The value of $\mathrm{k}$ is application-based and suggested to be $5 \%$ of the total pixel numbers in step 2.

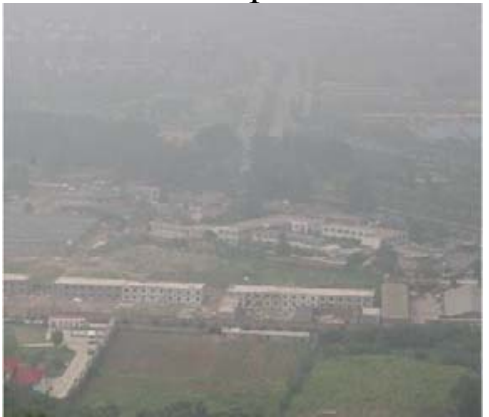

(a)Original image

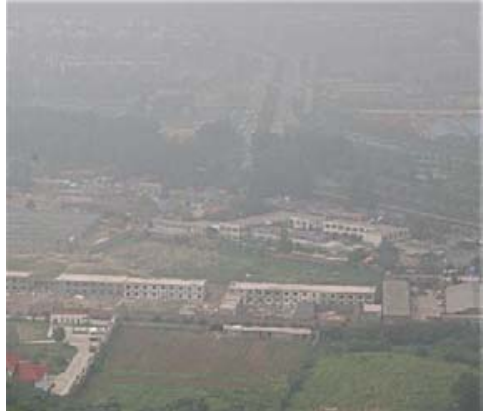

(b) Improve image after local contrast enhancement

Fig. 4. Comparison between original image and the image after local contrast enhancement

Fig. 4 indicates that the image after local contrast enhancement has higher definition.

Compared with other sharpening algorithm such as Laplace, USM et. al., local contrast enhancement makes the image show obvious processing mark. However, it improves the image definition more obviously than the sharpening algorithm, which can make the object in the image more clearly. This characteristic matches the demands of video monitoring.

\section{Experimental results}

In this section, the efficiency of the proposed image enhancement system will be presented and compared with He's method defined by [1]. All the experiments in this section are implemented on DM6467 Digital Media System-on-Chip.

To realize the image enhancement porting, our proposed image enhancement algorithm code should be inserted into the VIDECCOPY_TI_process function of Codec Engine on DSP side. The 
whole system control, such as program start and stop, menu selection, video acquisition and display, is implemented on ARM side. The ARM application consisted of six threads: main thread, video acquisition thread, video image processing thread, video display thread, video storage thread and mouse control thread. The system equipment is shown in Fig. 5.

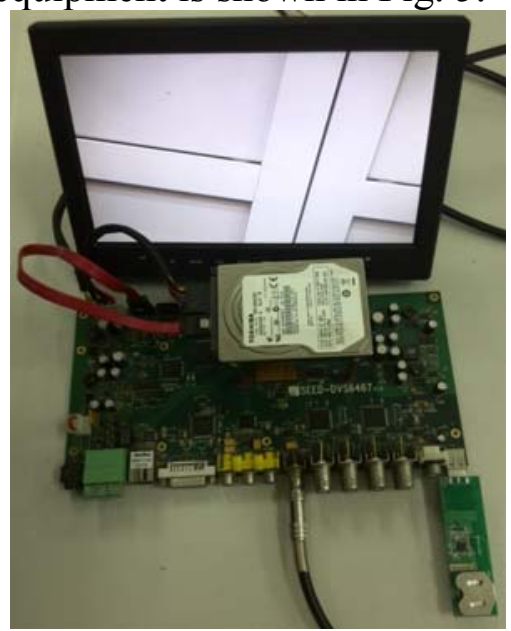

Fig. 5. The picture of the actual system based DM6467

Fig. 6 shows the interface of the video image enhancement system, which can switch between one channel video and four channel videos.

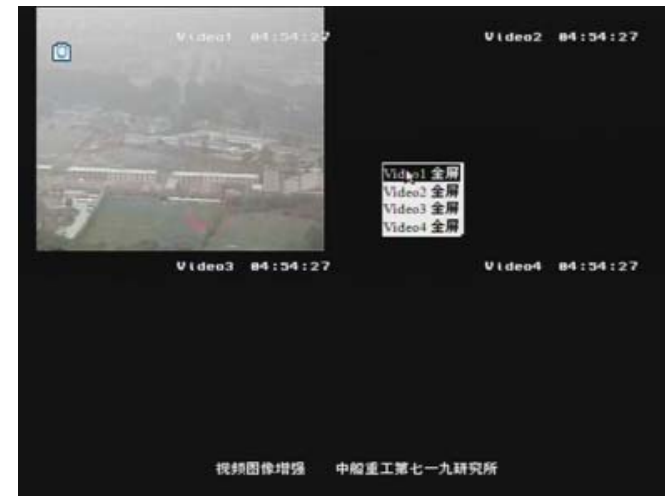

(a)Interface of four channel videos

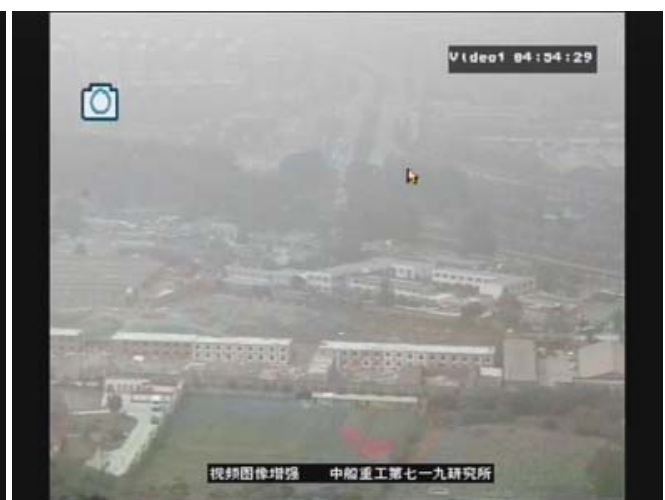

(b) Interface of one channel video

Fig. 6. The interface of the video image enhancement system

The Laplace evaluation function is chosen to evaluate the quality of the video. Laplace operator function is given in Eq. 12. The evaluation function, which equals to the sum of every pixel's Laplace operator, is proposed in Eq. 13. Finally, the image quality evaluation factor that is presented in Eq. 14 is used to evaluate the image quality. If the image is sharper, the value of $\mathrm{G}$ is bigger.

$$
\begin{aligned}
& \nabla^{2} f(x, y)=\left|\frac{\partial^{2} f(x, y)}{\partial x^{2}}\right|+\left|\frac{\partial^{2} f(x, y)}{\partial y^{2}}\right| \\
& S=\sum_{x}^{M} \sum_{y}^{N}[2 f(x, y)-f(x-1, y)-f(x+1, y)-f(x, y-1)-f(x, y+1)] \\
& G=\sqrt{S} /(M \times N)
\end{aligned}
$$

Two groups of original and improved image samples are given in Fig. 7. Table 1 shows the evaluation factor value of the image samples. It is obviously that the improved images are clear and distinctive. The evaluation factors of improved images increase noticeably. 

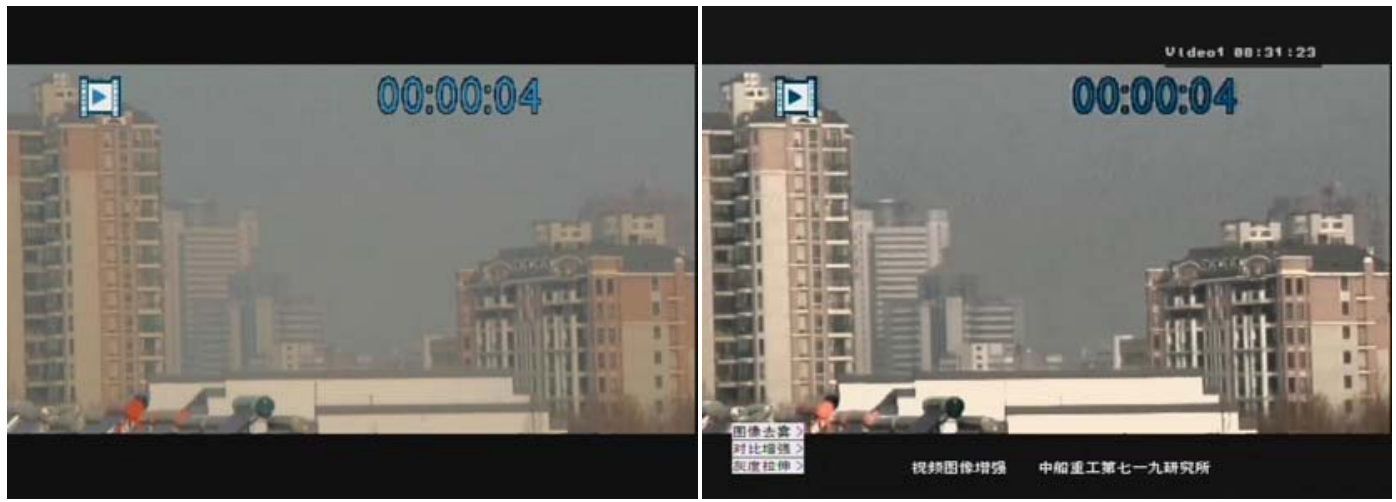

(a) One group of original and improved image sample

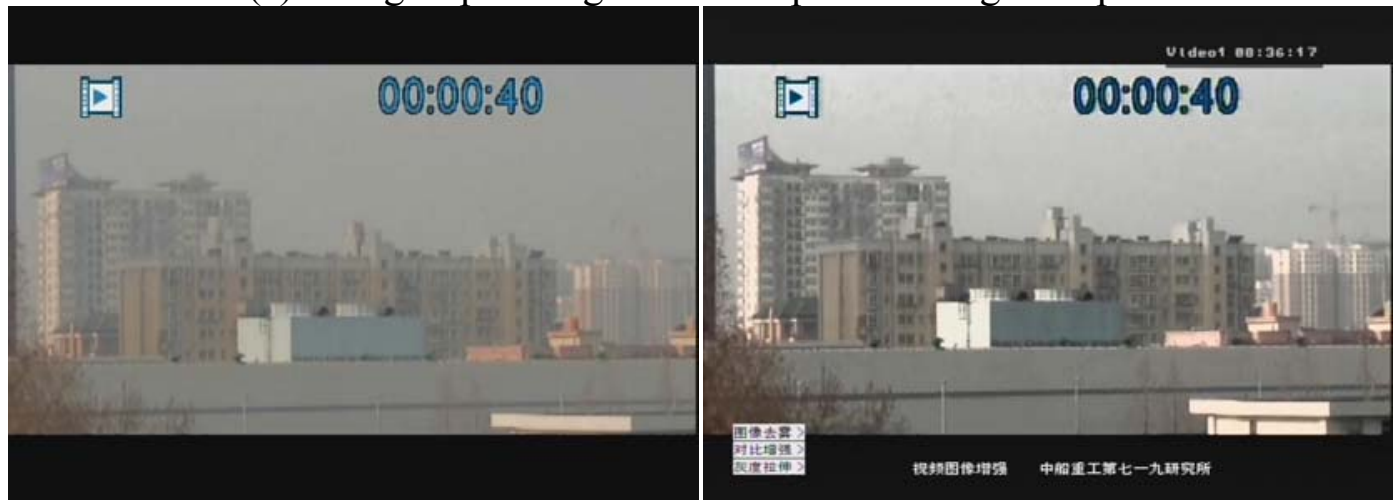

(b) The other group of original and improved image sample

Fig. 7. Two groups of original and improved image samples

Table 1.The evaluation factor value of the image samples

\begin{tabular}{c|c|c|c}
\hline Image samples & $\begin{array}{c}\text { The evaluation factor of } \\
\text { original images }\end{array}$ & $\begin{array}{c}\text { The evaluation factor of improved } \\
\text { images }\end{array}$ & $\begin{array}{c}\text { Improvement } \\
\text { rate }\end{array}$ \\
\hline 1 & 0.0105 & 0.0205 & $95.3 \%$ \\
\hline 2 & 0.0109 & 0.0208 & $89.98 \%$ \\
\hline
\end{tabular}

\section{Conclusion}

The dark channel prior based image dehazing algorithm is analyzed in the paper, firstly. In order to reduce block effects, the neighborhood similarity algorithm is introduced. For further improvement of the image quality, the Adaptive gray stretch algorithm and local contrast enhancement algorithm are proposed after image dehazing. Then, the video image enhancement system is fulfilled by applying the image enhancement algorithms on DM6467. Finally, the image enhancement experiment is presented to confirm the performance of the system. The image quality objective assessment shows that the system increases the image definition.

\section{References}

[1]. H. Kim, H. Jin, S. Hadap and I. Kweon, in: IEEE Conference on Computer Vision \& Pattern Recognition, Vol. 9, No. 4 (2013), p. 1460-1467

[2]. K. Kaur and N. Gupta, International Journal of Intelligent Systems \& Applications, Vol. 7, No. 5 (2015), p. 48-56.

[3]. Y. H.Shiau, P. Y.Chen, H. Y.Yang, C. H.Chen and S. S.Wang, Journal of visual communication and image representation, Vol. 25, No. 2 (2014), p. 445-453.

[4]. Y. C. Song, H. B. Luo, B. Hui, Z. Chang, in: Control and Decision Conference (2015), p. $5840-5845$

[5]. R. Gao, Y. Wang, M. Liu, X. Fan, Electronics Letters, Vol. 50, No. 24 (2014, p. 1826-1828 
[6]. F. Liu, C. Yang, in: IEEE International Conference on Signal Processing, Communications \& Computing, (2014), p. 483-486

[7]. A. Levin, D. Liscchinski, Y. Weiss, in: IEEE Conference on Computer Vision and Pattern Recognition, (2006), p. 61-68

[8]. H. J. Wang, G. Y. Wang and W. Ding, Control \& Automation, Vol. 27, No. 8 (2011), p. 166-167 